\title{
Foundations of the field theory: Connection of the field-theory equations with the equations of mathematical physics
}

\section{Petrova L.I.}

Moscow State University, Department of Computational Mathematics and Cybernetics, Moscow, Russia;

E-mail: Petrova <ptr@cs.msu.su>;

It is known that the equations of mathematical physics for material systems (material media) such as the thermodynamical, gas-dynamical, cosmologic systems, the systems of charged particles and others consist of the equations of conservation laws for energy, linear momentum, angular momentum, and mass.

It turns out that the mathematical physics equations for material systems possess some hidden properties that are manifested only under investigation the consistency of the conservation law equations. Under such investigation one obtains a relation in skew-symmetrical differential forms for the functionals such as the action functional, entropy, Pointing's vector, Einstein's tensor, wave function and other. As it is known, the field-theory equations, which describe physical fields, are equations for such functionals. And this emphasizes the correspondence between the field-theory equations and the relation obtained.

Such a relation, which appears to be evolutionary, nonidentical and self-varying, discloses the connection between the field-theory equations, which describe physical fields, and the equations of mathematical physics, which describe material media. The connection of the field-theory equations with the equations of mathematical physics and the correspondence between the field-theory equations and the evolutionary relation enables one to understand the basic principles of field theory and the properties of physical fields.

The present investigation was carried out with the help of skew-symmetric differential forms, which properties correspond to the conservation laws that lie at the basis of the equations of mathematical physics and the field-theory equations. In doing so, the skew-symmetric forms, which possess some peculiarities, were used. Namely, they are evolutionary ones and can generate closed exterior forms corresponding to the conservation laws for physical fields.

Keywords: material media, evolutionary relation, conservation laws, properties of the field theory.

DOI: $10.18698 / 2309-7604-2015-1-409-421$

\section{Introduction}

It is known that the equations of mathematical physics for material systems (material media) such as the thermodynamical, gas-dynamical, cosmologic systems, the systems of charged particles and others consist of the equations of conservation laws for energy, linear momentum, angular momentum, and mass. It turns out that the mathematical physics equations for material systems possess some hidden properties that are manifested only under investigation the consistency of the conservation law equations. Under such investigation one obtains a relation in skew-symmetrical differential forms [1] for the functionals such as the action functional, entropy, Pointing's vector, Einstein's tensor, wave function and other. As it is known, the field-theory 
equations, which describe physical fields, are equations for such functionals. And this emphasizes the correspondence between the field-theory equations and the relation obtained.

Such a relation, which appears to be evolutionary, nonidentical and self-varying, discloses the connection between the field-theory equations, which describe physical fields, and the equations of mathematical physics, which describe material media. The connection of the fieldtheory equations with the equations of mathematical physics and the correspondence between the field-theory equations and the evolutionary relation enables one to understand the basic principles of field theory and the properties of physical fields.

\section{Specific features of the equations of mathematical physics for material systems. Evolutionary relation}

The equations of mathematical physics for material systems, which consist of the equations of conservation laws for energy, linear momentum, angular momentum, and mass, possess the hidden properties, that enables to understand the basic principles of field theory. Such properties are due to the specific features of equations of mathematical physics, which become evident when studying the consistency equations of conservation laws.

\subsection{Analysis of consistency of the conservation law equations. Evolutionary relation for the state functionals}

The consistency of the conservation law equations is realized under correlation of the conservation law equation between themselves.

Let us analyze the correlation of the equations that describe the conservation laws for energy and linear momentum.

We introduce two frames of reference: the first is an inertial one (this frame of reference is not connected with the material system), and the second is an accompanying one (this frame of reference is connected with the manifold built by the trajectories of the material system elements).

The energy equation in the inertial frame of reference can be reduced to the form:

$$
\frac{D \psi}{D t}=A_{1}
$$

where $D / D t$ is the total derivative with respect to time, $\psi$ is the functional of the state that specifies the material system, $A_{1}$ is the quantity that depends on specific features of material system 
and on external energy actions onto the system. [The action functional, entropy, wave function can be regarded as examples of the functional $\psi$. Thus, the equation for energy presented in terms of the action functional $S$ has a similar form: $D S / D t=L$, where $\psi=S, A_{1}=L$ is the Lagrange function. In mechanics of continuous media the equation for energy of an ideal gas can be presented in the form [2]: $D s / D t=0$, where $s$ is the entropy.]

In the accompanying frame of reference the total derivative with respect to time is transformed into the derivative along the trajectory. Equation of energy is now written in the form

$$
\frac{\partial \psi}{\partial \xi^{1}}=A_{1}
$$

Here $\xi^{1}$ is the coordinate along the trajectory.

In a similar manner, in the accompanying reference system the equation for linear momentum appears to be reduced to the equation of the form

$$
\frac{\partial \psi}{\partial \xi^{v}}=A_{v}, \quad v=2, \ldots
$$

where $\xi^{v}$ are the coordinates in the direction normal to the trajectory, $A_{v}$ are the quantities that depend on the specific features of material system and on external force actions.

Eqs. (1) and (2) can be convoluted into the relation

$$
d \psi=A_{\mu} d \xi^{\mu}, \mu=1, v
$$

Relation (3) can be written as

$$
d \psi=\omega
$$

here $\omega=A_{\mu} d \xi^{\mu}$ is the skew-symmetrical differential form of the first degree. (A summing over repeated indices is carried out.)

In the general case (for energy, linear momentum, angular momentum and mass) this relation will be the written as 


$$
d \psi=\omega^{p}
$$

where $\omega^{p}$ is the form degree $p$ ( $p$ takes the values $p=0,1,2,3$ ).

[A concrete form of relation (4) and its properties in the case of the Euler and Navier-Stokes equations were considered in papers [3]. In this case the functional $\psi$ is the entropy $s$. A concrete form of relation (5) for $p=2$ were considered for electromagnetic field in paper http://arxiv.org/pdf/math-ph/0310050v1.pdf.]

Since the conservation law equations are evolutionary ones, the relations obtained are also evolutionary relations, and the skew-symmetric forms $\psi$ and $\omega^{p}$ are evolutionary ones.

\subsection{Properties of evolutionary relation}

The evolutionary relation obtained possesses inconvenient properties. This relation appears to be nonidentical and self-varying. [Such properties relate to the fact that this relation includes a skew-symmetric form, which, unlike the exterior skew-symmetric form, is defined on nonintegrable deforming manifold and is evolutionary one. (About the properties of such skew-symmetric form one can read, for example, in papers [1,3].]

Evolutionary relation proves to be nonidentical since the differential form in the right-hand side of this relation is not a closed form, and, hence, this form cannot be a differential like the lefthand side.

To justify this we shall analyze relation (4).

The form $\omega=A_{\mu} d \xi^{\mu}$ is not a close form since its differential is nonzero. The differential $d \omega$ form $\omega=A_{\mu} d \xi^{\mu}$ can be written as $d \omega=K_{\alpha \beta} d \xi^{\alpha} d \xi^{\beta}$, where $K_{\alpha \beta}=A_{\beta: \alpha:}-A_{\alpha: \beta}$ are the components of the commutator of the form $\omega$, and $A_{\beta: \alpha:}, A_{\alpha: \beta}$ are the covariant derivatives. If we express the covariant derivatives in terms of the connectedness (if it is possible), then they can be written as $A_{\beta: \alpha:}=\partial A_{\beta} / \partial \xi^{\alpha}+\Gamma_{\beta \alpha}^{\sigma} A_{\sigma}$, where the first term results from differentiating the form coefficients, and the second term results from differentiating the basis. We obtain the following expression for the commutator components of the form $\omega$ :

$$
K_{\alpha \beta}=\left(\frac{d A_{\beta}}{\partial \xi^{\alpha}}-\frac{d A_{\alpha}}{\partial \xi^{\beta}}\right)+\left(\Gamma_{\beta \alpha}^{\sigma}-\Gamma_{\alpha \beta}^{\sigma}\right) A_{\sigma}
$$


(In the commutator of exterior form, which is defined on integrable manifold, the second term is absent.) The coefficients $A_{\mu}$ of the form $\omega$ have been obtained either from the equation of the conservation law for energy or from that for linear momentum. This means that in the first case the coefficients depend on the energetic action and in the second case they depend on the force action. In actual processes energetic and force actions have different nature and appear to be inconsistent. Therefore the first member of the commutator is nonzero. The expressions $\left(\Gamma_{\beta \alpha}^{\sigma}-\Gamma_{\alpha \beta}^{\sigma}\right)$ entered into the second term are just components of commutator of metric form that specifies the manifold deformation and hence equals nonzero. It turns out that the differential of the form $\omega$ is nonzero. Thus, the form $\omega$ to be unclosed and cannot be a differential like the lefthand side. This means that the evolutionary relation cannot be an identical one.

The evolutionary nonidentical relation is a selfvarying one, since this relation includes two objects one of which appears to be nonmeasurable.

\section{Hidden properties of the equations of mathematical physics for material media: the existence of double solutions}

The evolutionary relation discloses a peculiarity of the equations of mathematical physics, namely, the existence of double solutions. The evolutionary relation was obtained when studying the consistency of the conservation law equations. The nonidentity of the evolutionary relation points to the fact that the conservation law equations appear to be inconsistent. This means that the initial set of equations of mathematical physics proves to be nonintegrable (it cannot be convoluted into identical relation for differentials and be integrated). That is, the solutions to the mathematical physics equations are not functions (they will depend on the commutator of the form $\omega^{p}$ ). This also points to the fact that the tangent manifold, on which the solutions are defined, is not integrable.

During selfvariation of evolutionary relation the conditions when an inexact (closed on pseudostructure) exterior form is obtained from evolutionary form can be realized. This leads to the fact that from nonidentical evolutionary relation it will be obtained an identical relation, and this and this will point out to a consistency of the conservation law equations and an integrability of the mathematical physics equations.

However, the transition from unclosed evolutionary form (with nonzero differential) to closed exterior form (with vanishing differential) is possible only as degenerate transformation, namely, a transformation that does not conserve the differential. 
The degenerate transformation can take place under additional conditions. The conditions of degenerate transformation can be due to the degrees of freedom of material system (such as the translational degrees of freedom, internal degrees of freedom of the system elements and etc.). [The conditions of degenerate transformation are reduced to vanishing of such functional expressions as determinants, Jacobians, Poisson's brackets, residues and others.]

If the conditions of degenerate transformation are realized, from the unclosed evolutionary form $\omega^{p}$ (see evolutionary relation (5)) with non vanishing differential one can obtain a closed (only on some pseudostructure) exterior form with vanishing (interior) differential. That is, it is realized the transition

$$
d \omega^{P} \neq 0 \rightarrow d_{\pi} \omega^{P}=0, d_{\pi}^{*} \omega^{P}=0
$$

The realization of the conditions $d_{\pi} \omega^{p}=0, d_{\pi}{ }^{*} \omega^{p}=0$ means that it is realized a closed dual form ${ }^{*} \omega^{p}$, which describes some structure $\pi$ (which is a pseudostructure with respect to its metric properties), and it the closed exterior (inexact) form $\omega_{\pi}^{p}$, which basis is a pseudostructure, is obtained. On an pseudostructure, from evolutionary relation (5) it follows the relation

$$
d \psi_{\pi}=\omega_{\pi}^{p}
$$

which occurs to be an identical one, since the form $\omega_{\pi}^{p}$ is a differential. The identity of the relation obtained from the evolutionary relation means that on the pseudostructure the equations of conservation laws become consistent. This points out to that the equations of mathematical physics become locally integrable (only on pseudostructure). Pseudostructure in this case is integrable structure. The solutions to the mathematical physics equations on integrable structures are generalized solutions, which are discrete functions, since they are realized only under additional conditions (on the integrable structures).

\subsection{Physical meaning of double solutions to the equations of mathematical physics. Description of the state of material medium}

The evolutionary relation can describe the material medium state, so how this relates includes the state functional, which specifies the material system state.

But here there is some delicate matter. Although the evolutionary relation includes the state functional (which specifies the material medium state), but since this relation is nonidentical one, 
from this relation one cannot get the differential of the state functional $d \psi$. This points out to the absence of the state function and means that the material medium is in the non-equilibrium state. The non-equilibrium means that an internal force acts in material medium. It is evident that the internal force is described by the commutator of skew-symmetric form $\omega^{p}$. (Everything that gives a contribution into the commutator of evolutionary form $\omega^{p}$ leads to emergence of internal forces that causes the non-equilibrium state of material medium (see [4]).) The solutions of the first type, which are not functions (since they depend on the commutator of form $\omega^{p}$, describe such a non-equilibrium state of material medium.

Another property of the nonidentical evolutionary relation, namely, its selfvariation, points out to the fact that the non-equilibrium state of material medium turns out to be selfvarying. State of material medium changes but in this case remains to be non-equilibrium during this process, since the evolutionary relation remains to be nonidentical during the process of selfvariation.

The realization of identical relation from the evolutionary one point out to the transition of material medium to a localli equilibrium state.From identical relation one can define the differential of the state functional, and this points out to a presence of the state function and the transition of material medium from non-equilibrium state into equilibrium one. However, such a state of material medium turns out to be realized only locally due to the fact that differential of the state functional obtained is an differential interior (only on pseudostructure). And yet the total state of material medium remains to be non-equilibrium state because the evolutionary relation, which describes the material medium state, remains nonidentical one. (That is, there exists a duality. Nonidentical evolutionary relation goes on to act simultaneously with identical relation.) It may be noted that these results show that the functionals of evolutionary relation are actually state functionals.]

The transition from non-equilibrium state to locally equilibrium state means that unmeasured quantity, which is described by the commutator and act as internal force, converts into a measured quantity of material medium. This reveals in emergence of some observed formations in material medium. Waves, vortices, fluctuations, turbulent pulsations and so on are examples of such formations. The intensity of such formations is controlled by a quantity accumulated by the evolutionary form commutator.

Thus, from the evolutionary relation it follows that the equations of mathematical physics has double solutions that describe the transition of a material medium from non-equilibrium state into a localli equilibrium one, and this process is accompanied by origination of a discrete (observable) formation. Such a process is connected with origination of physical structures. 


\subsection{Differential-geometrical structures and their physical meaning. Physical structures}

As it was shown, the discrete realization of generalized solution (and an advent of observable formation) is related with the realization of dual form and closed inexact exterior form. The closed dual form and associated closed inexact exterior form made up a differentialgeometrical structure that describes a pseudostructure with conservative quantity (a closed dual form describes a pseudostructure, and a closed exterior form, as it is known, describes a conservative quantity, since the differential of closed form is equal to zero). Such a differentialgeometrical structure possesses a duality. In the case of the equations of mathematical physics, such a differential-geometrical structure is an integrable structure, on which the solutions to the mathematical physics equations become functions, that is, a generalized solutions. (The structures like the characteristics, singular points, characteristic and potential surfaces,are such integrable structures.) The differential-geometrical structure is associated with a discrete observable formation, to which the generalized solution is assigned.

On the other hand, the differential-geometrical structure describes a conservative object (a pseudostructure with conservative quantity). It appears that the physical structures that made up physical fields are such conservative objects. (In the next section the justification of this statement will be presented.)

\section{Connection of the field-theory equation with the equations of mathematical physics for material media}

The evolutionary relation discloses one more unique property of the equations of mathematical physics, namely, a connection between the mathematical physics equations, which describe material media, and the field-theory equations, which describe physical fields. And this emphasizes the correspondence between the field-theory equations and the evolutionary relation. Such correspondence bases on the properties of conservation laws. The peculiarity consists in the fact that the equations of mathematical physics consist of the equations of conservation laws for energy, linear momentum, angular momentum, and mass, which are conservation laws for material media, whereas the field-theory equation are based on conservation laws for physical fields, that claim an existence of conservative quantities or objects. The conservation laws for physical fields are described by closed exterior skew-symmetric forms. (The Noether theorem is an example.) It turns out that there exists a connection between the conservation laws for material media and the conservation laws for physical fields. This follows from the evolutionary relation. And this discloses a connection between the field-theory equations and the equations of mathematical physics. 


\subsection{Basis of the field-theory equations: Closed exterior forms correspond to conservation laws for physical fields}

From the closure conditions for exterior differential form $d \theta^{k}=0$ one can see that the closed exterior differential form is a conservative quantity. This means that the closed exterior differential form can correspond to conservation law for physical fields, namely, to existence of a certain conservative physical quantity. If form is a closed inexact one then from conditions of closure for the dual form $d_{\pi}{ }^{*} \theta^{k}=0$ (describing the pseudostructure) and closed inexact form $d_{\pi} \theta^{k}=0$ (describing the conservative quantity) one can see that the dual form and inexact form describe a conservative object that can also correspond to conservation law for physical fields.

The equations of existing field theories, which describe physical fields, are the equations obtained on the basis of the properties of exterior form theory.

Closed inexact exterior or dual forms are solutions of the field-theory equations. And there is the following correspondence.

-Closed exterior forms of zero degree correspond to quantum mechanics.

-The Hamilton formalism bases on the properties of closed exterior and dual forms of first degree.

-The properties of closed exterior and dual forms of second degree are at the basis of the equations of electromagnetic field.

-The closure conditions of exterior and dual forms of third degree form the basis of equations for gravitational field.

One can see that field theory equations connected with closed exterior forms of a certain degree. This enables one to introduce a classification of physical fields in degrees of closed exterior forms. Such a classification shows that there exists an internal connection between field theories that describe physical fields of various types. It is evident that the degree of closed exterior forms is a parameter that integrates field theories into unified field theory [1]. (A significance of exterior differential forms for field theories consists in the fact that they disclose the properties that are common for all field theories and physical fields irrespective of their specific type. This is a step to building a unified field theory.)

It appears that the closed exterior and dual forms, on which the field-theory equations are based, are generated by the evolutionary relation, obtained from the equations of mathematical physics. This is justified by the fact that there is a correspondence between the field-theory equations and the evolutionary relation. 


\subsection{Correspondence between the field-theory equations and the evolutionary relation}

The field-theory equations, which describe physical fields, are equations for functionals such as wave function, the action functional, Pointing's vector, Einstein's tensor, and others. The nonidentical evolutionary relations derived from the equations of mathematical physics, which describe material media, are relations for all these functionals.

To the connection between the field-theory equations and the equations of mathematical physics it also points out to the fact that, all equations of field theories, as well as the evolutionary relation, are nonidentical relations in differential forms or in the forms of their tensor or differential (i.e. expressed in terms of derivatives) analogs. For example,

- the Einstein equation is a relation in differential forms;

- the Dirac equation relates Dirac's bra- and cket - vectors, which made up a differential form of zero degree;

- the Maxwell equations have the form of tensor relations;

- the Schr\H\{o\}dinger's equations have the form of relations expressed in terms of derivatives and their analogs.

From the evolutionary relation one can obtain closed inexact exterior forms which corresponds to the conservation laws for physical fields and on which (as it was shown) the field theory bases.

From the field-theory equations, as well as from the nonidentical evolutionary relation, the identical relation, which contains the closed exterior form, is obtained. As one can see, from the field-theory equations it follows such identical relation as

- the Poincare invariant, which connects closed exterior forms of first degree;

- the relations $d \theta^{2}=0, d^{*} \theta^{2}=0$ are those for closed exterior forms of second degree obtained from Maxwell equations;

- the Bianchi identity for gravitational field.

Thus, one can see that there exists a correspondence between the field-theory equations, which describe physical fields, and the evolutionary relation obtained from the equations of mathematical physics for a material medium.

Such a correspondence between the evolutionary relation and the field-theory equations point to a connection of the field-theory equations and the equations of mathematical physics for material media. 


\subsection{Hidden properties of the field theory}

Connection of the field-theory equation with the equations of mathematical physics for material media enables one to understand the basic principles of field theory and the properties of physical fields.

1. From the correspondence between the field-theory equations and the evolutionary relation it follows that the functionals of field-theory equations (such as wave function, the action functional, the Pointing vector, Einstein tensor, and others) are functionals that specify the state of relevant material medium (material system), that is, they are state functionals (functionals that describe a state of relevant material medium).

2. Closed inexact exterior and dual forms, which are solutions to the field-theory equations and corresponds to conservation laws for physical fields, are generated by evolutionary form obtained from the conservation law equations for material systems, namely, the conservation laws for energy, linear momentum, angular momentum, and mass. This points to a relation of the conservation laws for physical fields (availability of conserved quantities or objects) and the conservation laws for material media (the conservation laws for energy, linear momentum, angular momentum, and mass).

3. The degree of closed exterior forms, which can serve as a parameter of unified field theory is connected with the number of the equations of interacting noncommutative conservation laws for material systems.

4. The constants and characteristics of field theories are connected with characteristics of relevant material media. (But this connection is indirect. This connection is realized in evolutionary process).

5. Physical structures that obey the conservation law for physical fields (existence of conservative objects) and made up physical fields are described by the differential-geometrical structures obtained from the equations of mathematical physics for material media.

6. Type of physical structures generated by material media depends on the degree $p$ of the evolutionary form in evolutionary relation connected with the number of interacting conservation laws, on the degree $k$ of the closed exterior form obtained, and on the dimension $n$ of the initial inertial space.

7. Realization of the differential-geometrical structures (inexact exterior skew-symmetric forms and relevant dual forms, which describe physical structures) proceeds discretely under realization of the degrees of freedom of material systems. This explains the quantum character of field theories. 
8. One can see the correspondence between the degree $k$ of the closed forms realized and the type of interactions. Thus, $k=0$ corresponds to strong interaction, $k=1$ corresponds to weak interaction, $k=2$ corresponds to electromagnetic interaction, and $k=3$ corresponds to gravitational interaction.

The connection between physical structures and closed exterior and dual forms allows to disclose the properties and the characteristics of physical fields.

Conserved physical quantity (closed exterior form) describes a certain charge. Under transition from some structure to another, the conserved on pseudostructure quantity, which corresponds to the closed exterior form, changes discretely, and the pseudostructure changes discretely as well.

The discrete changes of the conserved quantity and pseudostructure are determined by the value of the evolutionary form commutator, which is a commutator at the time when the physical structure emerges. The first term of the evolutionary form commutator obtained from the derivatives of the evolutionary form coefficients controls the discrete change of the conserved quantity. The second one obtained from the derivatives of the metric form coefficients of the initial manifold controls the pseudostructure change. Spin is the example of the second characteristic.

[As it was shown, a non-measurable quantity that is accumulated in the evolutionary form commutator partly converts into conservative quantity of physical (observed) structures under realization of any degrees of freedom (to which a degenerate transformation is assigned).

A non-measurable quantity that does not convert into physical structure turns out to be a non-observed and non-measurable quantity. Dark matter and dark energy are such an essence which reveals as the result of the noncommutativity of conservation laws of relevant material media produced by various nonpotential actions. The deformation of manifolds, which is made up by the trajectories of the material system elements, arisen under the action of external forces (due to nonpotential actions) relates to this fact.]

[In paper [5] was carried out proof of the invariance of the equations for the energy, momentum and continuity. But Einstein did not put the question of the consistency of these equations. The presence of energymomentum tensor in Einstein's equation supposes that the equations of conservation laws of energy and momentum are consistent identically. But, as has been shown, the consistence of conservation law equations (due to noncommutativity of conservation laws) is fulfilled only discretely. In particular, this means that the energymomentum tensor is fulfilled only discretely. And this imposes restrictions on the Einstein equation.]

\section{References}

1. Petrova L.I. (2008). Exterior and evolutionary differential forms in mathematical physics: Theory and Applications.

2. Clark J.F., Machesney M. (1964). The Dynamics of Real Gases. Butterworths, London. 
3. Petrova L.I. (2013). A new mathematical formalism: Skew-symmetric differential forms in mathematics, mathematical physics and field theory. Moscow: URSS.

4. Petrova L.I. (2014). Hidden properties of the Navier-Stokes equations. Double solutions.

Origination of turbulence, Theoretical Mathematics and Applications. Scienpress Ltd, Vol.4, No.3, 91-108.

5. Einstein A. (1953) The Meaning of Relativity. Princeton. 